To the Editors:

\title{
The first successful liver transplant in Sri Lanka
}

\author{
Ceylon Medical Journal 2011; 56: 81-82
}

In the absence of liver transplantation, end stage liver disease carries a very poor prognosis in Sri Lanka. Currently, living donor transplantation abroad is the only chance for survival, but is financially prohibitive to most. We report the first successful liver transplant in Sri Lanka.

A 49-year old, $50 \mathrm{~kg}$ man with endstage liver disease was referred to the Colombo University Surgical Unit for liver transplantation in March 2010. He had haematemesis and encephalopathy having had worsening ascites in the previous two months. This previously alcoholic man had been abstinent since July 2009.

Examination showed a shrunken liver and the spleen was palpable $6 \mathrm{~cm}$ below the left costal margin. Routine haematology and biochemistry confirmed impairment of both liver and renal functions. His serum virology (Hepatitis A, B, C and HIV) was negative and tumour markers (alfa-fetoprotein, carcinoembryonic antigen, CA19/9) were not significantly elevated. Triphasic CT excluded hepatocellular carcinoma and confirmed portal vein patency.

The Model for End-stage Liver Disease (MELD) derives a disease severity score (6-40) predicting the 90 day mortality and is used for prioritising patients on the transplant waiting list [1]. In this instance the MELD score $[3.8 \times \log (\mathrm{e})$ (bilirubin, $2.6 \mathrm{mg} / \mathrm{dL})+11.2 \times \log (\mathrm{e})(\mathrm{INR}, 2)+$ $9.6 \log (\mathrm{e})$ (creatinine, $1.8 \mathrm{mg} / \mathrm{dl}$ )] was 23 with a predicted 90 day mortality of $35 \%$, a strong justification for liver transplantation. Furthermore, he was psychologically stable with adequate social support making him a suitable candidate.

Being unable to find a suitable living donor and bear the high financial cost for liver transplantation abroad, he consented to be the first liver recipient in Sri Lanka. While awaiting a donor liver, he experienced an episode of spontaneous bacterial peritonitis, encephalopathy and six episodes of hepatorenal syndrome. He was managed with peritoneal paracentesis, antibiotics, human albumin, saltfree diet, diuretics, beta blockers, proton pump inhibitors, vitamin $\mathrm{K}$ and lactulose.

On 26 June 2010, we were informed of a previously well 41-year old road accident victim who was 'brain stem dead' from head injuries. He had no truncal injuries and was haemodynamically stable. The blood group was compatible and virology negative. His family consented for organ donation.

Having obtained the necessary legal authorisation, we proceeded to retrieve the liver using histidine, tryptophan, ketoglutarate (Cutodial ${ }^{\circledR}$, HTK) cold preservative solution on 27 June 2010. The recipient operation commenced shortly after, under general anaesthesia with invasive monitoring. The cirrhotic liver was explanted and the whole donor liver was transplanted orthotopically.

The donor vena cava was anastomosed in a 'piggy back' fashion. End to end anastomoses were carried out for the portal vein, hepatic artery and the common bile duct in that order. The total anhepatic time was 150 minutes. The liver perfused well and haemodynamic stability was maintained with transfusion of 28 packs each of red cells, plasma and platelets.

Immunosuppression was induced with methylprednisolone and basiliximab and maintained with prednisolone, mycophenolate mofetil and tacrolimus. Postoperative recovery was complicated by staphylococcal septicaemia which responded to antibiotics. The liver enzymes and INR declined after 24 hours and normalised within the first week. Serial sonography confirmed good perfusion and biliary drainage of the graft. However, transaminases rose on day 20 and a biopsy confirmed mild rejection (Banff score 4) [2]. This was successfully treated with methylprednisolone pulsing. The transaminases, bilirubin, albumin and INR were all normal when he was sent home on day 28.

He remains well with normal liver biochemistry and sonography 8 months after transplantation on low dose maintenance immunosuppression and has returned to full time employment.

Although kidney transplantation has been practiced in Sri Lanka since 1985 [3] current facilities for liver transplantation are inadequate in our hospitals. Availability of modern facilities for intra-operative autotransfusion ('Cell Saver') and coagulation monitoring (thromboelastography) would have significantly reduced transfusion requirements and minimised risks to our patient $[4,5]$. If we are to provide a regular liver transplantation service that fulfills the needs of our population, dedicated up to date facilities are required.

\section{References}

1. Kamath PS, Wiesner RH, Malinchoc M, et al. Model to predict survival in patients with end stage liver disease. Hepatology 2001; 33: 464-70.

2. Banff schema for grading liver allograft rejection: an international consensus document. Hepatology 1997; 25: 658-63. 
3. Sheriff R, Sheriffdeen AH, Jayasekare G, et al. The first kidney transplantation in Sri Lanka. Ceylon Medical Journal 1987; 32: 15-27.

4. Sankarankutty AK, Teixeira AC, Souza FF, et al. Impact of blood salvage during liver transplantation on reduction in transfusion requirements. Acta Cirurgica Brasileira 2006; 21 (Sup 1): 44-7.

5. Wang SC, Shieh JF, Chang KY, et al. Thromboelastographyguided transfusion decreases intraoperative blood transfusion during orthotopic liver transplantation: randomized clinical trial. Transplant Proceedings 2010; 42: 2590-3.

\section{S M Wijeyaratne ${ }^{1}$, N Gunawansa ${ }^{1}$, M R N Cassim ${ }^{1}$, A Abayadeera ${ }^{1}$}

${ }^{1}$ Department of Surgery, University of Colombo, Sri Lanka.

Correspondence: SMW, e-mail <mandika59@hotmail.com>. Received 29 October 2010 and revised version accepted 24 February 2011. Competing interests: none declared. 